\title{
Focused Sonography in Detecting Hemoperitoneum in Blunt Abdominal Trauma Patients, Correlation with Computed Tomography
}

\author{
Nauman Al Qamari ${ }^{1}$, Raza Sayani ${ }^{1, ~}$, Muhammed Ali ${ }^{2}$, Muhammad Tamim Akhtar ${ }^{2}$ \\ ${ }^{1}$ Radiology Department, Aga Khan University hospital, Karachi, Pakistan \\ ${ }^{2}$ Radiology Department, Ziauddin University Hospital, Karachi, Pakistan
}

\section{Email address:}

nauman.alqamari@aku.edu(N.Al Qamari),sayani_raza@yahoo.com(R. Sayani),docali80@hotmail.com(M. Ali), profmtakhtar@live.com(M. T. Akhtar)

To cite this article:

Nauman Al Qamari, Raza Sayani, Muhammed Ali, Muhammad Tamim Akhtar. Focused Sonography in Detecting Hemoperitoneum in Blunt Abdominal Trauma Patients, Correlation with Computed Tomography, International Journal of Medical Imaging.

Vol. 1, No. 1, 2013, pp. 7-11. doi: 10.11648/j.ijmi.20130101.12

\begin{abstract}
To determine the accuracy of focused assessment with sonography for trauma in detecting the hemoperitoneum in blunt abdominal trauma patients and comparing it with Computed Tomographic findings. The study was conducted at Radiology department of Dr. Ziauddin University Hospital from March 2010 to September 2010. 140 patients presented with complaints of acute blunt abdominal trauma. Focused assessment with sonography for trauma was performed. Presence of intra-abdominal fluid was taken as positive finding for hemoperitoneum. Then all the patients underwent computed tomography of abdomino-pelvic region. Focused assessment with sonography for trauma findings were compared with computed tomography results. In 83 patients, the FAST showed free intra abdominal fluid and was taken as 'positive'. Out of these 83 cases 80 (96\%) were 'true positive' while the remaining 3(4\%) were 'false positive' when compared with results of CT scan.Similarly, in 57 patients, no free intra abdominal fluid was detected on FAST and they were considered 'negative'. From these 57 patients, $50(88 \%)$ patients were 'true negative' and $7(12 \%)$ were 'false negative' because intra abdominal free fluid was seen in them on subsequent CT scan. Thus the sensitivity and specificity of FAST in detecting intra abdominal free fluid was calculated to be $91.9 \%$ and $94.34 \%$ respectively. FAST scan is moderately sensitive in detecting intra abdominal free fluid when compared with the results of CT scan.
\end{abstract}

Keywords: Blunt Abdominal Trauma, Focused Assessment with Sonography for Trauma, Computed Tomography, Accuracy, Hemoperitoneum

\section{Introduction}

Trauma related injuries continue to be a worldwide problem. It is the leading cause of death from ages $15-44$ in the developed countries, and an ever increasing cause of death and disability in the developing world. In fact, traumatic injury may soon outpace infectious diseases as a leading cause of worldwide mortality.[1]

Abdominal injuries rank third as a cause of traumatic death just after head and chest injuries . [2] Historical records are full of description of blunt abdominal trauma victims who turned pale, cold and died without overt evidence of hemorrhage. Such undetected hemoperitoneum was one of the most common causes of death in injured patients. Unrecognized abdominal injuries are frequently the cause of preventable death.

The abdomen of trauma victims is routinely evaluated with physical examination and clinical signs that have relatively low diagnostic accuracy $(47 \%$ to $87 \%)$, especially when the patient had a decreased consciousness level, neurological deficit, other associated injuries, or was under the influence of drugs or medications ${ }^{2}$. Diagnostic peritoneal lavage (DPL), ultrasound (FAST), and computed tomography (CT) are typical tests used for abdominal evaluation in trauma. .[3,4]

Diagnostic peritoneal lavage has disadvantage of being invasive technique, hence it is not suitable for conscious traumatic patients and in pediatric patients. .[5] Ultrasound has become a standard of care in most emergency departments. Bedside ultrasonography provides non- 
invasive, readily available, and time-saving option for patients with blunt abdominal trauma. [6,7] The term 'focused abdominal sonography for trauma' (FAST) scan has been used since the early 1990s. Nowadays, FAST is the standard practice for blunt abdominal trauma in most emergency departments in developed world.

Computed Tomography scan of the abdomen and pelvis is the procedure of choice to evaluate the hemodynamically stable patient who has sustained blunt or penetrating trauma. CT has replaced Diagnostic Peritoneal Lavage (DPL) as the first method of choice in many trauma centers worldwide $[8,9]$. However, it has several disadvantages as it is relatively expensive, involves the usage of ionizing radiation and requires the shifting of patient to the scanner which may interfere with ongoing resuscitation. Furthermore, it is not always available particularly in small peripheral centres. $[5,6,10]$.

\section{Objective}

To determine the accuracy of focused assessment with sonography for trauma in detecting the hemoperitoneum in blunt abdominal trauma patients and comparing it with Computed Tomographic findings.

\section{Materials \& Methods}

This is a Cross sectional study, it was carried out at radiology department of Dr. Ziauddin University Hospital from March 2010 to September 2010. All patients with recent blunt abdominal trauma due to road vehicle accident, fall or violence within last 24 hours who presented to the radiology department were included. Those excluded from the study were patients who presented more than 24 hours after blunt abdominal trauma, who had penetrating abdominal injuries, who were suffering from preexisting disease in which there was possibility of presence of intra abdominal fluid or those with history of recent abdominal surgery

Patient size was calculated by placing the following values in the formula: Expected sensitivity $0.86^{4}$, expected specificity $0.95^{4}$, expected prevalence $0.17^{15}$, desired precision 0.12, confidence level 0.90. Sampling Technique was non probability, purposive.

History was taken in each case about the mechanism and duration of trauma, preexisting disease and previous surgical history. Informed consent was taken from all the patients after explaining the purpose, procedure and risks \& benefits. Approval from the ethical committee of our hospital was taken.

Focused assessment with sonography for trauma was performed on SSD-4000 plus (ALOKA, Japan) with a $5 \mathrm{MHz}$ convex transducer. Intra abdominal free fluid was searched in perihepatic and perisplenic regions, Morrison's pouch, both para-colic gutters, reteroperitoneum and in pelvis. Presence of intra-abdominal fluid was taken as positive finding for hemoperitoneum. US results were recorded on a written form..

Then all the patients subsequently underwent computed tomography of abdomino pelvic region. Computed tomography was performed on multi slice ASTEION 4 (Toshiba, Japan) typically within 30 minutes after FAST. Field of examination was from the lung bases to the pelvis with $5 \mathrm{~mm}$ contiguous sections and a table speed of $5 \mathrm{~mm} / \mathrm{sec}$ (pitch). CT was routinely performed after contrast material had been injected intravenously as a powerinjected bolus of $140 \mathrm{ml}$ of $240 \mathrm{mg}$ of iodine per milliliter at a rate of $3 \mathrm{ml} / \mathrm{sec}$. A uniphasic injection of intravenous contrast material with a scan delay of 60 seconds was used. No oral contrast was given. FAST findings were compared with computed tomography results.

All information was documented with the help of proforma.

\section{Data Analysis Procedure}

All collected information was entered into SPSS version 10 and analyzed through it. Male to female ratio and mean standard deviation for age distribution were computed. Graphs and charts regarding gender, mechanism of trauma and time lapse between trauma and Focused assessment with sonography for trauma were obtained. A $2 \times 2$ table was constructed and sensitivity, specificity, positive predictive value, negative predictive value and accuracy of Focused assessment with sonography for trauma in detecting hemoperitoneum in blunt abdominal trauma were calculated by using computed tomography findings.

\section{Results}

One hundred and forty patients who fulfilled the inclusion criteria were included in the study. There were 90 $(64.29 \%)$ males and $50(35.71 \%)$ females. The ages of these patients ranged b/w 1 year and 89 years. The mean age was 33 yrs. Mean ages in male and female patients were not significantly different.

Mechanism of injury was motor vehicle accident in 64 patients ( $45 \%$ ), fall from height in 43 patients ( $30 \%$ ), assault in 18 patients $(13 \%)$, pedestrian struck in 8 patients $(6 \%)$ and other causes like during playing game in 7 patients $(5 \%)$. Most of the patients included in the study presented within first 12 hrs while about $50 \%$ presented within $6 \mathrm{hrs}$ of occurrence of injury. It is shown in chart (1)

$\begin{array}{lrcc}\text { Within } 1 \mathrm{hr} & 5 & 6-12 \mathrm{hrs} & 40 \\ 1-3 \mathrm{hrs} & 12 & 12-24 \mathrm{hrs} & 25 \\ 3-6 \mathrm{hrs} & 58 & \end{array}$

Out of 140 patients, in 83 patients the FAST scanning showed free intra abdominal fluid and was taken positive. The most common site where free fluid was detected was pelvis and then perihepatic area. In 57 (40\%) patients, free fluid was localized in a single region while in other patients more than one pocket of free fluid was found. In 51 patients ( $36 \%$ ) free intra abdominal fluid was detected in pelvis , in 46 patients $(33 \%)$ it was seen in perihepatic region, in 37 
( $26 \%$ ) it was localized in perisplenic region and in 23 patients ( $16 \%$ ) free fluid was seen in paracolic gutters. Figure 1 and 2 .In 5 patients $(<1 \%)$ hemo pericardium was detected.

From those 83 FAST positive patients, $80(96 \%)$ were true positive while $3(4 \%)$ were false positive and showed no free fluid on consecutive CT scan.

False positive results may be due to distended, fluid filled bowel loops. In one female patient of child bearing age, free fluid was detected in cul - de - sac in $18^{\text {th }}$ day of menstrual cycle and it was considered to be physiological and taken as negative.

FAST scan was unable to detect free intra abdominal fluid in 57 patients and they were taken as FAST 'negative'. Out of these 57 cases, 49 ( $86 \%)$ correlated well with the CT scan findings. The remaining $8(14 \%)$ showed free intra abdominal fluid on CT scan which was failed to detect on FAST scan.
Two other patients were having free fluid due to preexisting medical disorder. One was cirrhotic liver with portal hyper tension and ascites. In other patient, CT scan confirmed the presence of free fluid in the spleeno-renal angle, but also showed peri portal and peri cholecystic fluid; these findings were considered non - traumatic for the purpose of this study, as they may be due to third spacing of the fluid related to vigorous fluid resuscitation rather than injury.

The overall sensitivity of FAST was $90.9 \%$ and specificity was $94.23 \%$ in this study. Similarly the positive predictive value (PPV) was calculated as $96.38 \%$ and negative predictive (NPV) as $85.96 \%$ in this study.

Detection of organ injury was beyond the scope of this study, however during interpretation of CT scan about 95 patients $(68 \%)$ were having organic injuries. Out of these 95 patients, 69 were having grade $1 \& 2$ liver / splenic injuries which were managed non - surgically.

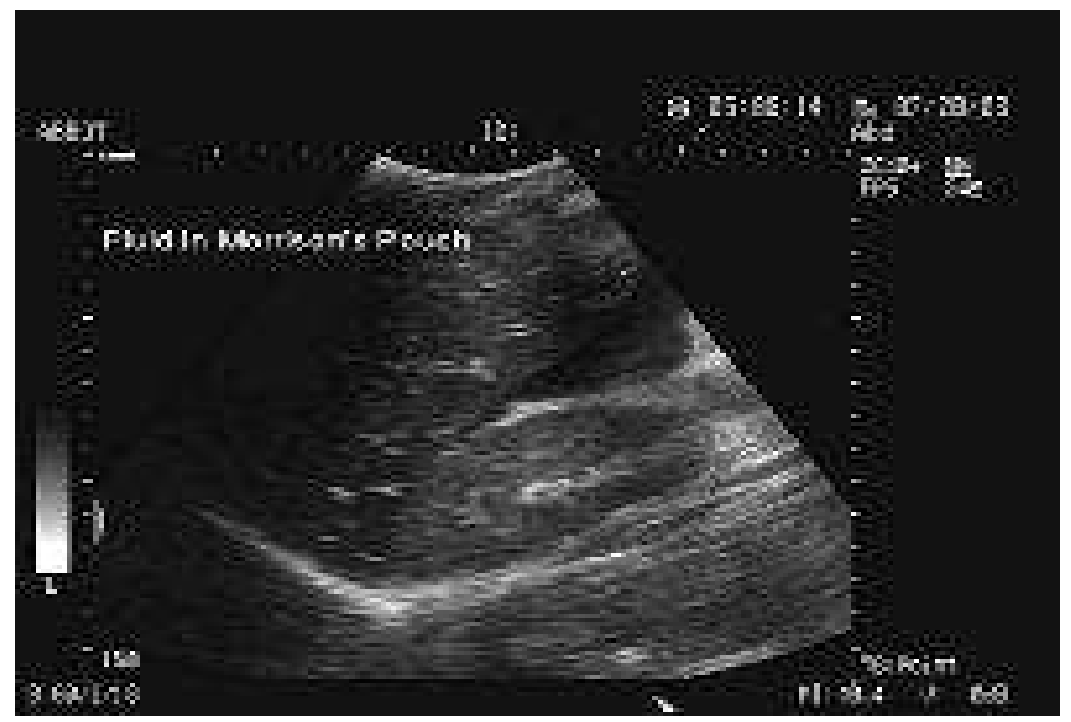

Figure 1. Blunt abdominal trauma. Free fluid in the Morison pouch.

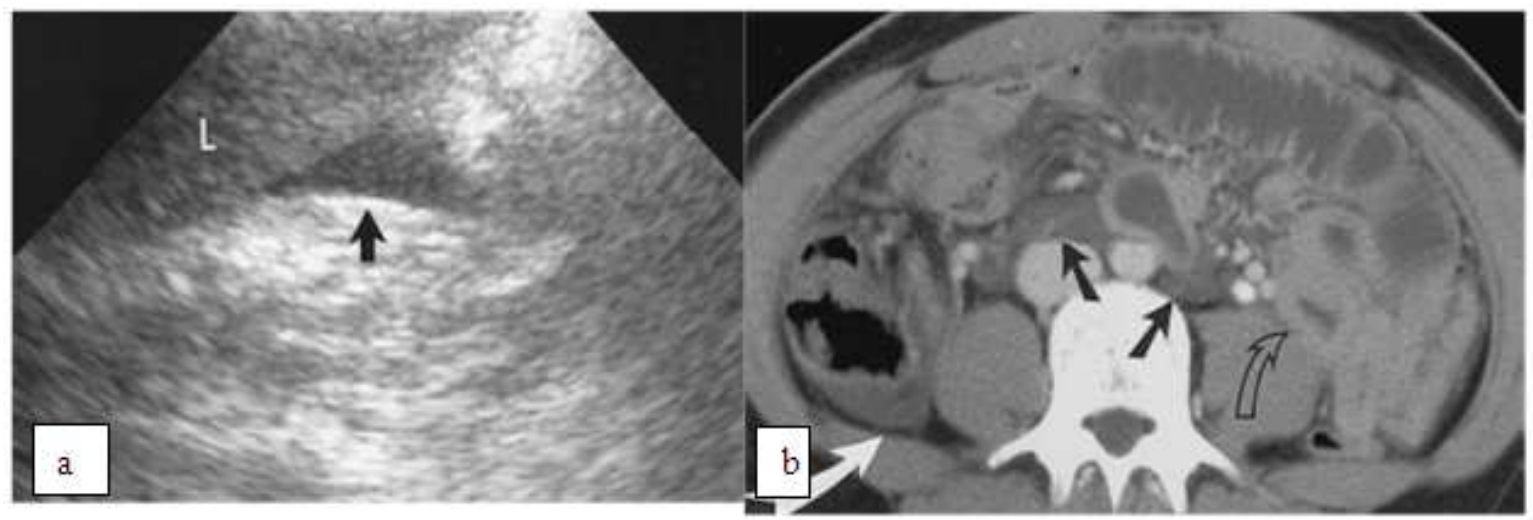

Figure 2. Images in a 19-year-old woman involved in a motor vehicle accident. (a) Longitudinal screening US image shows a small amount of fluid (arrow) near the inferior liver (L) edge. (b) Contrast-enhanced transverse abdominal CT image confirms bowel injury with thickened jejunum (open arrow) and fluid in the mesentery (black solid arrows) and right paracolic gutter (white solid arrow).

\section{Discussion}

Abdominal injuries are the third common cause of traumatic death after head and chest injuries[11]. Diagnostic peritoneal lavage (DPL) has been done 
historically to detect hemoperitoneum. The introduction of bedside ultrasonography provides an accurate, non invasive, readily available and time saving tool for triage of patient with blunt abdominal trauma. In this study we focused on the accuracy of FAST scan in the detection of hemoperitoneum resulting from blunt abdominal trauma. Further this study also addresses the utility of sonography for identifying injury to specific organs.

Hemodynamically stable patients with positive FAST results may require a $\mathrm{CT}$ scan to better define the nature and extent of their injuries. Taking every patient with a positive FAST result to the operating room may result in an unacceptably high laparotomy rate.[12]

Hemodynamically stable patients with negative FAST results require close observation, serial abdominal examinations, and a follow-up FAST examination. However, strongly consider performing a CT scan, especially if the patient is intoxicated or has other associated injuries.[13]

Hemodynamically unstable patients with negative FAST results are a diagnostic challenge to the treating physician. Options include DPL, exploratory laparotomy, and, possibly, a CT scan after aggressive resuscitation.[14,15]

In previous studies, many investigators have reported high sensitivity of ultrasound in detecting hemoperitoneum in BAT patients. In 1989 Gruessner and co-workers compared sonography with DPL findings in 808 patients. The sensitivity and specificity of ultrasonography was reported as $89 \%$ and $100 \%$ respectively. [16] In a prospective study of 800 patients, Healey calculated the sensitivity of ultrasonography of $88 \%$ and specificity of $98 \%$ with an overall accuracy of $97 \%$.[17]

In late 1990s Bode analyzed 1,671 consecutive sonogram and determined that ultrasonography had a sensitivity of $88 \%$, specificity of $100 \%$ and an overall accuracy of $99 \% .[18]$

In a more recent study Lingawi and Buckley evaluated 1,090 patents using focused abdominal sonography FAST protocol. Patients with negative sonogram underwent 12 hours of clinical observation followed by repeat ultrasound or computed tomography (CT). Using this approach, their reported sensitivity and specificity of sonography were $94 \%$ and $98 \%$ respectively.[19] In 2001, researcher at university of California evaluated 3,264 patient of blunt abdominal trauma with sonography and reported sonography sensitivity of $60 \%$ in detecting hemoperitoneum, The specificity and accuracy were $98 \%$ and $94 \%$ respectively. Luke et al examined 259 children admitted for blunt abdominal trauma who underwent ultrasound and their calculated sensitivity, specificity, and accuracy for detection of hemoperitoneum were $89 \%, 96 \%$ and $94 \%$ respectively.[20]

These studies suggest that sonography is sensitive and highly specific for the detection of hemoperitoneum in BAT patients. However few researchers have reported decreased sensitivity of sonography. Coley and co-workers compared US with CT and determined that the sensitivity and specificity of US relative to CT were $55 \%$ and $83 \%$ respectively, and they concluded that US was not accurate enough to replace CT in this setting.[21] In our study sensitivity of sonography is $90.9 \%$ and specificity is $94 \%$. This is comparable with the study of several previous authors. The present results confirmed that FAST ultrasound is a sensitive and specific diagnostic tool in the detection of hemoperitoneum in BAT patient.

Some authors have suggested that serial sonogram obtained over time as the peritoneal cavity fills with blood may improve the accuracy of sonography in detecting hemoperitoneum. Siniluoto et al detected 2 of 5 splenic injuries with initial sonography and identified all 5 injuries 24 hours later using follow up ultrasound.[22] Similarly Handerson and colleagues reported 4 cases in which patients' initial sonogram were negative for hemoperitoneum and follow up sonogram obtained on serial sonography were positive.[23]Poletti and coworker found that sensitivity improved at second ultrasound by 15\%.[24] Almost similar results were found in present study in which 8 patients showed free intra-abdominal fluid after few hours on serial ultrasound. These results suggest that in patients with high clinical suspicion of abdominal injury, a repeat ultrasound should be done after few hours interval to detect small occult hemoperitoneum.

Solid organs injuries may results in hemoperitoneum that may not be detected on ultrasound.

Detection of solid organ injury requires more efforts and skill than simply searching for free fluid. Some authors have addressed the potential of ultrasound for the detection of acute solid injuries. Poletti et al has found that adding analysis of parenchymal lesions to analysis of free fluid improved the sensitivity of FAST ultrasound by only $7 \%$ compared with the analysis of free fluid alone (from $65 \%$ to 72\%).[24] Our results are also in agreement with Poletti, which showed only $2.4 \%$ increase ( 2 in 85 patients) in sensitivity after including solid organs assessment along with hemoperitoneum.

As abdominal trauma is a dynamic event, scanning too early might miss a significant abdominal injury as free intra peritoneal fluid need time of accumulate. These results are comparable to Tsui CL and colleagues.[25] This can be demonstrated by one result in which sonographic sensitivity rose from $60 \%$ within 1 hour after BAT to $97 \%$ in $6-12$ hours after injury as shown in graph.

There were two false positive cases after CT scan in this study. Causes include fluid in cul-de-sac due to ovulation, fluid in the stomach or dilated bowel and visualization of perinephric fat. Almost similar causes were described by several authors.

\section{Limitations}

Our study is hampered by certain limitations. First of all the severity of hemodynamic instability of patient was not assessed in our study. Similarly, by focusing only at the detection of hemoperitoneum, we could not successfully 
triage patient for surgery and laparotomy after sonographic assessment and these decisions were usually made after CT scanning.

\section{Conclusion}

We conclude that FAST scan is a useful diagnostic tool in the initial assessment of blunt abdominal trauma patients. It is accurate with high specificity and positive predictive value. The high negative predictive value makes it a useful screening tool for detecting hemoperitoneum.

Serial sonography can improve overall sensitivity of FAST scan and should be done in all patients with high clinical suspicion of visceral injury and negative early ultrasound.

Ultrasound is limited mainly by its low sensitivity in directly demonstrating solid organs injuries.

\section{Refrences}

[1] Krug EG, Sharma GK, Lozano R: The global burden of injuries. Am J Public Health 2000, 90:523-526.

[2] Perry JF. A Five-year survey of 152 acute abdominal injuries J Trauma 1965; 5:53-57.

[3] Elton C, Riaz AA, Young N, Schamschula R, Papadopoulos B, Malka V. Accuracy of computed tomography in the detection of blunt bowel and mesenteric injuries. Br J Surg 2005;92:1024-8

[4] Ochsner MG, Knudson MM, Pachter HL, Hoyt DB, Cogbill TH. Significance of minimal or no intraperitoneal fluid visible on $\mathrm{CT}$ scan associated with blunt liver and splenic injuries: a multicenter analysis. J Trauma 2000;49:505-10.

[5] Nural MS, Yarden T, Guven H, Baydin A, Bayrak IK, Kati C Diagnostic value of $\mathrm{u} / \mathrm{s}$ in evaluation of blunt abdominal trauma. Diagn Interv Rad 2005;11:41-4.

[6] Kristensen JR, Bueman B, Keuhl E. Ultrasonic scanning in the diagnosis of splenic haematomas. Acta Chir Scand 1971;137:653-657

[7] Kirkpatrick AW. Clinician-performed focused sonography for the resuscitation of trauma. Crit Care Med 2007;35:S162-72.

[8] Bakker J, Gender R, Mali W, Leenen L. Sonography as the primary screening method in evaluating blunt abdominal trauma. Journal of Clinical Ultrasound 2005;33:155-63.

[9] Tso P, Rodriguez A, Cooper C, Militello P, Mirvis S, Badellino $\mathrm{MM}$, et al. Sonography in blunt abdominal trauma: a preliminary progress report. J Trauma. Jul 1992;33(1):39-43; discussion 43-4

[10] Soundappan SV, Holland AJ, Cass DT, Lam A. Diagnostic accuracy of Surgeon performed focused abdominal sonography (FAST) in blunt pediatric trauma.
Injury2005;36:970-5.

[11] Farahmand N, Sirlin CB, Brown MA, Shragg GP, Fortlage D, Hoyt DB et al. Hypotensive patients with blunt abdominal trauma. Performance of screening ultrasound. J Radiology 2005;235:436-43.

[12] Richards JR, Schleper NH, Woo BD, Bohnen PA, McGahan JP. Sonographic assessment of blunt abdominal trauma: a 4-year prospective study. J Clin Ultrasound 2002; 30:5967.3

[13] Griffin XL, Pullinger R. Are diagnostic peritoneal lavage or focussed abdominal sonography for trauma safe screening investigations for hemodyamically stable patients after blunt abdominal trauma. A review of the literature. J Trauma 2007;62:779-84

[14] Sirlin CB, Brown MA, Andrade-Barreto OA. Blunt abdominal trauma: clinical value of negative screening US scans. Radiology 2004; 230:661-668.

[15] Stengel D, Bauwens K, Sehouli J. Systematic review and meta-analysis of emergency ultrasonography for blunt abdominal trauma. Br J Surg. 2001; 88: 901-912

[16] Gruessner R, Mentges B, Duber CH, Ruckert K, Rothmund M. Sonography versus peritoneal lavage in blunt abdominal trauma. J Trauma 1989;29:242-4.

[17] Healey MA, Simons RK, Winchell RJ. A prospective evaluation of abdominal ultrasound in blunt trauma: is it useful? J Trauma 1996;40:875-83.

[18] Bode PJ, Edwards MJ, Kruit MC, Van Vugt AB. Sonography in a clinical algorithm for early evaluation of 1671 patients with blunt abdominal trauma. AJR Am J Roentgenol 1999; 172:905-911.

[19] Lingawi S, Buckley A. Focused abdominal US in patients with trauma. Radiology 2000; 217:426-429.

[20] Luks FI, Lemire A, St-Vil D, Di Lorenzo M, Filiatrault D, Ouimet A. Blunt abdominal trauma in children: the practical value of ultrasonography. J Trauma 1993;34:607-10.

[21] Coley BD, Mutabagani KH, Martin HC, Zumberge N, Cooney DR, Caniano DA et al. Focused abdominal sonography for trauma (FAST) in children with blunt abdominal trauma. The Journal of Trauma 2000;40(5):902-5.

[22] Siniluoto TM, Paivansalo MJ, Lanning FP, Typpo AB, Lohela PK, Kotaniemi AE. Ultrasonography in traumatic splenic rupture. Clin Radiol 1992;46:391-6.

[23] Henderson SO, Sung J, Mandavia D. Serial abdominal ultrasound in the setting of trauma. J Emerg Med 2000;18:79-81.

[24] Poletti PA, Kinkel K, Vermeulen B, et al. Blunt abdominal trauma: should US be used to detect both free fluid and organ injuries? Radiology. 2003; 227:95-103.

[25] Tsui CL, Fung HT, Chung KL, Kam CW. Focused abdominal sonography for trauma in the emergency department for blunt abdominal trauma. Int $\mathrm{J}$ Emerg Med2008;1:183-7. 